\title{
Monocyte-suppressing effect of high-dose metformin in fenofibrate-treated patients with impaired glucose tolerance
}

\author{
Robert Krysiak, Anna Gdula-Dymek, Bogusław Okopień \\ Department of Internal Medicine and Clinical Pharmacology, Medical University of Silesia, Medyków 18, \\ PL 40-752 Katowice, Poland \\ Correspondence: Robert Krysiak, e-mail: r.krysiak@interia.pl
}

\begin{abstract}
:
Background: Fibrates were found to reduce cytokine release and low-grade inflammation in patients with impaired glucose tolerance. The aim of this study was to investigate whether these effects of fibrates may be potentiated by metformin treatment.

Methods: The study included 43 patients with isolated impaired glucose tolerance and normal plasma lipids who had been treated for at least 6 months with micronized fenofibrate ( $200 \mathrm{mg}$ daily). These subjects were randomly assigned to 12 weeks' treatment with either high dose metformin ( $3 \mathrm{~g}$ daily in three divided doses) or placebo. Plasma lipids, glucose homeostasis markers, monocyte cytokine release and plasma $\mathrm{C}$-reactive protein levels were determined before randomization and at the end of the treatment.

Results: Metformin treatment reduced plasma C-reactive protein levels and monocyte release of tumor necrosis factor- $\alpha$ and interleukin-6, as well as tended to reduce monocyte release of interleukin-1 $\beta$ and monocyte chemoattractant protein-1, which was accompanied by an improvement in insulin sensitivity.

Conclusions: Our results show that high-dose metformin produces monocyte-suppressing and systemic anti-inflammatory effects in fibrate-treated patients with isolated impaired glucose tolerance. This suggests that fibrate-metformin combination therapy may bring clinical benefits to impaired glucose tolerance patients at high cardiovascular risk.
\end{abstract}

Key words:

fibrates, inflammatory cells, low-grade inflammation, metformin, prediabetes

\begin{abstract}
Abbreviations: CRP - C-reactive protein, $\mathrm{HDL}$ - high density lipoproteins, hsCRP - high sensitivity C-reactive protein, HOMA-IR - the homeostasis model assessment of insulin resistance, IGT - impaired glucose tolerance, LDL - low density lipoproteins, MCP-1 - monocyte chemoattractant protein-1, TNF- $\alpha$ - tumor necrosis factor- $\alpha$
\end{abstract}

\section{Introduction}

Recent results from our laboratory suggest that fenofibrate, particularly administered together with met- formin, produces pluripotential pleiotropic effects, including a reduction in systemic inflammation and monocyte cytokine release, as well as the improvement in insulin sensitivity and hemostasis in type 2 diabetic patients with mixed dyslipidemia $[4,11]$. Similarly, administered to metabolic syndrome patients, fenofibrate not only improved glucose homeostasis (a decrease in fasting and post-challenge plasma glucose, insulin resistance and glycated hemoglobin), but also produced $\mathrm{C}$-reactive protein (CRP)-lowering and monocyte-suppressing effects [3]. In the subgroup of patients with impaired glucose tolerance 
(IGT), monocyte-suppressing effects of fenofibrate were more prominent than the analogous effects of atorvastatin [3].

Although administration of metformin with fenofibrate may be considered an alternative treatment option in subjects in whom statin therapy is either contraindicated or results in adverse effects [11], to the best of our knowledge, to date, no study has assessed the benefits of fibrate-metformin combination therapy in patients with prediabetes. Therefore, in this study we assessed whether the addition of metformin potentiates the effects of chronic fenofibrate treatment on monocyte secretory function and systemic inflammation. Monocytes are the primary inflammatory cell type that infiltrate early atherosclerotic plaques, playing a variety of roles in atherosclerotic plaque development and its clinical sequelae $[1,16]$. The high dose of metformin used in this study ( $\mathrm{g}$ daily) is considered safe and effective and is commonly used in the clinical practice [13].

\section{Materials and Methods}

The study population consisted of 43 subjects (20-70 years old) with isolated IGT and normal lipid profile who had been complying with lifestyle intervention and had been treated with micronized fenofibrate at the daily dose of $200 \mathrm{mg}$ for at least 6 months before the beginning of the study. At the beginning of the study, fenofibrate-treated patients were compared with the control group including 23 age-, sex- and weight-matched normolipidemic subjects with isolated IFG who had been receiving metformin (3 $\mathrm{g}$ daily) and following lifestyle modification for more than 6 months but had not been treated with any hypolipidemic agent. Isolated IGT was diagnosed when fasting plasma glucose was less than $100 \mathrm{mg} / \mathrm{dl}$, and plasma glucose concentration $2 \mathrm{~h}$ after a 75 -g oral glucose load was at least $140 \mathrm{mg} / \mathrm{dl}$ but less than $200 \mathrm{mg} / \mathrm{dl}$. Normal lipid profile was defined as total plasma cholesterol below $200 \mathrm{mg} / \mathrm{dl}$, LDL cholesterol less than $130 \mathrm{mg} / \mathrm{dl}$ and triglycerides below $150 \mathrm{mg} / \mathrm{dl}$. The study protocol was approved by our institutional review board, and subjects gave written, informed consent to participate in the study. Fenofibrate-treated subjects were randomly assigned to 12 weeks' treatment with either high dose metformin (3 g daily in three divided doses) or placebo. Throughout the entire study period, all participants continued to comply with lifestyle modifications and were treated with the same dose of fenofibrate. Compliance, investigated during each visit, was regarded as satisfactory if the number of tablets taken by a patient ranged from $90 \%$ to $110 \%$.

Venous blood samples were taken in the fasting state in the morning both on the day of allocation and after 12 weeks of treatment. To minimize analytical errors, all assays were conducted in duplicate by a person unaware of the patient's data. Lipid profile, plasma insulin, as well as fasting and $2-\mathrm{h}$ postchallenge plasma glucose levels were assayed by routine laboratory techniques. The homeostasis model of insulin resistance (HOMA-IR) index was calculated as [fasting serum glucose $(\mathrm{mg} / \mathrm{l}) \times$ fasting serum insulin $(\mu \mathrm{U} / \mathrm{ml}) / 405]$, with lower values indicating a higher degree of insulin sensitivity. Total non-estrified free fatty acids (FFA) were determined by an enzymatic assay using reagents from Alpha Laboratories (Eastleigh, Hants, UK). Plasma levels of CRP were measured using a highsensitivity monoclonal antibody assay (hsCRP) (MP Biomedicals, Orangeburg, NY). Unstimulated and lipopolysaccharide-stimulated monocytes were cultured in triplicate and cytokine release were measured as described earlier [7, 9]. Because unstimulated monocytes from both control and fenofibrate-treated patients released hardly detectable amounts of tumor necrosis factor- $\alpha$ (TNF- $\alpha)$, interleukin-1 $\beta$, interleukin-6 and monocyte chemoattractant protein-1 (MCP-1), only monocyte release from stimulated monocytes was included in the final analyses. The minimum detectable levels for the assessed cytokines were: $4.4 \mathrm{pg} / \mathrm{ml}$ (TNF- $\alpha$ ), $1.0 \mathrm{pg} / \mathrm{ml}$ (interleukin-1 $\beta$ ), $3.8 \mathrm{pg} / \mathrm{ml}$ (interleukin-6) and $5.0 \mathrm{pg} / \mathrm{ml}$ (MCP-1). The intra- and interassay coefficients of variation for the assessed markers were less than 5 and $9 \%$, respectively.

The Shapiro-Wilk test was used to assess the distribution of variables. In the case of variables with nonnormal distribution (HOMA-IR, hsCRP, cytokines), $\log$ transformation was used to fit a normal distribution curve. Between-group comparisons were performed using one-way ANOVA followed by the post-hoc Bonferroni test (when three groups were compared) or using independent samples $t$-test (when two groups were compared). Student's paired $t$-test was used to evaluate differences in the measured variables within the same treatment group. Correlations between the study parameters were calculated using Kendall's $\tau$ test. Statistical significance was assumed at $\mathrm{p}<0.05$. Analyses were conducted using GraphPad Prism 2.01 software and Statistica 6.1. 


\section{Results}

At baseline, both groups of fenofibrate-treated patients and the control group were comparable with respect to sex distribution, age, body weight, medical background, clinical characteristics and baseline laboratory results (Tab. 1). Compared to activated monocytes from fenofibrate-treated patients, lipopolysaccharide-stimulated monocytes from control subjects produced larger amounts of TNF- $\alpha$, interleukin-1 $\beta$, interleukin-6 and MCP-1 and had higher plasma levels of hsCRP (Tab. 1).
Fenofibrate + metformin and fenofibrate + placebo treatment were well tolerated and all but one patient completed the study. This patient (receiving both fenofibrate and metformin) was withdrawn from the study because of diarrhea and vomiting. All laboratory safety tests remained within normal limits throughout the treatment period. Twelve-week fenofibrate + placebo treatment produced no effect on lipid profile, glucose homeostasis markers, hsCRP and monocyte cytokine release (Tab. 2). Fenofibrate + metformin reduced plasma hsCRP levels, inhibited TNF- $\alpha$ and interleukin- 6 release, as well as tended to reduce interleukin- $1 \beta(\mathrm{p}=0.065)$ and MCP-1 release $(p=0.069)($ Tab. 1). Except for an insignificant de-

Tab. 1. Baseline characteristics of patients ${ }^{1}$

\begin{tabular}{|c|c|c|c|}
\hline & Fenofibrate + Placebo & Fenofibrate + Metformin & Control group \\
\hline Number of patients & 20 & 22 & 23 \\
\hline Age [years; mean (SD)] & $51(4)$ & $50(4)$ & $49(5)$ \\
\hline Women [\%] & 35 & 36 & 39 \\
\hline Smokers $[\%]$ & 25 & 32 & 30 \\
\hline Body mass index $\left[\mathrm{kg} / \mathrm{m}^{2} ;\right.$ mean (SD)] & $28.3(2.2)$ & $28.5(2.6)$ & $27.9(2.8)$ \\
\hline Waist circumference [cm; mean (SD)] & $104(7)$ & $103(8)$ & $103(7)$ \\
\hline Systolic blood pressure [mmHg] & $132(7)$ & $134(7)$ & $130(8)$ \\
\hline Diastolic blood pressure [mmHg] & $86(4)$ & $87(4)$ & $85(4)$ \\
\hline Coronary artery disease $[\%]$ & 20 & 18 & 17 \\
\hline Arterial hypertension [\%] & 35 & 32 & 26 \\
\hline Fasting glucose [mg/dl; mean (SD)] & $90(5)$ & $92(4)$ & $90(4)$ \\
\hline 2-h post-glucose load plasma glucose [mg/dl; mean (SD)] & $164(11)$ & $159(10)$ & $161(10)$ \\
\hline HOMA-IR [mean (SD)] & $3.2(0.5)$ & $3.0(0.4)$ & $2.8(0.4)$ \\
\hline Free fatty acids $\mu \mathrm{mol} / /$; mean (SD)] & $290(43)$ & $285(39)$ & $301(48)$ \\
\hline Total cholesterol [mg/dl; mean (SD)] & $168(16)$ & $172(14)$ & $165(15)$ \\
\hline LDL cholesterol [mg/dl; mean (SD)] & $102(12)$ & $104(15)$ & $101(10)$ \\
\hline HDL cholesterol [mg/dl; mean (SD)] & $48(4)$ & $49(4)$ & $50(4)$ \\
\hline Triglycerides [mg/dl; mean (SD)] & $125(14)$ & $123(12)$ & $121(14)$ \\
\hline hsCRP [mg/l; mean (SD)] & $2.2(0.5)^{\star \star}$ & $2.0(0.5)^{\star \star}$ & $3.1(0.5)$ \\
\hline TNF- $\alpha$ release $[\mathrm{pg} / \mathrm{ml}$; mean (SD)] & $808(102)^{\star \star}$ & $823(112)^{\star \star}$ & $1128(183)$ \\
\hline Interleukin-1 $\beta$ release $[\mathrm{pg} / \mathrm{ml}$; mean (SD)] & $85.2(12.1)^{\star \star}$ & $82.1(11.0)^{\star * *}$ & $111.5(12.8)$ \\
\hline Interleukin-6 release [ng/ml; mean (SD)] & $6.1(0.8)^{\star \star \star}$ & $6.2(0.9)^{\star \star}$ & $8.4(1.1)$ \\
\hline MCP-1 release [ng/ml; mean (SD)] & $14.2(1.8)^{\star *}$ & $14.6(2.0)^{*}$ & $18.5(2.3)$ \\
\hline
\end{tabular}

\footnotetext{
${ }^{1}$ Only data of subjects who completed the study were included in the final analyses. ${ }^{\star} p<0.05,{ }^{* \star} p<0.01,{ }^{* \star *} p<0.001$ vs. control group
} 
crease in plasma triglycerides $(p=0.086)$, plasma lipids in fenofibrate + metformin-treated patients remained at the similar level throughout the study (data not shown). This treatment also reduced HOMA-IR, FFA and 2-h postchallenge plasma glucose levels. Compared to monocytes of fenofibrate + placebotreated subjects, monocytes of IGT patients receiving fenofibrate + metformin treatment released less amounts of all cytokines measured and exhibited lower plasma levels of hsCRP.
On the day of allocation, plasma hsCRP correlated with monocyte cytokine release ( $\mathrm{r}$ values between 0.51 and $0.65, \mathrm{p}<0.001$ ). Moreover, hsCRP and cytokine release correlated with HOMA-IR ( $\mathrm{r}$ values between 0.47 and $0.60, p<0.001$ ), and with FFA ( $\mathrm{r}$ values between 0.35 and $0.42, p<0.01$ ). There was a correlation between metformin treatment-induced changes in hsCRP and the changes in monocyte secretory function ( $\mathrm{r}$ values between 0.43 and $0.58, \mathrm{p}<$ 0.001). The effect of metformin treatment on hsCRP

Tab. 2. Effect of metformin on glucose homeostasis, plasma hsCRP and monocyte cytokine release in fenofibrate-treated patients with impaired glucose tolerance ${ }^{1}$

\begin{tabular}{|c|c|c|}
\hline Variable & $\begin{array}{c}\text { Fenofibrate + Placebo } \\
\text { Mean (SD) }[\Delta \%]\end{array}$ & $\begin{array}{c}\text { Fenofibrate }+ \text { Metformin } \\
\text { Mean (SD) }[\Delta \%]\end{array}$ \\
\hline \multicolumn{3}{|l|}{ HOMA-IR } \\
\hline Before randomization & $3.2(0.5)$ & $3.0(0.4)$ \\
\hline After 12 weeks of treatment & $3.1(0.5)[-3]$ & $1.9(0.3)[-37]]^{\star \star \star \# \# \# ~}$ \\
\hline \multicolumn{3}{|l|}{ Free fatty acids $[\mu \mathrm{mol} / \mathrm{l}]$} \\
\hline Before randomization & $290(43)$ & $285(39)$ \\
\hline After 12 weeks of treatment & $279(40)[-4]$ & $204(37)[-28]^{\star \star \#}$ \\
\hline \multicolumn{3}{|l|}{ Fasting glucose [mg/dl] } \\
\hline Before randomization & $90(5)$ & $92(4)$ \\
\hline After 12 weeks of treatment & $91(4)[1]$ & $88(4)[4]$ \\
\hline \multicolumn{3}{|c|}{ 2-h post-glucose load plasma glucose [mg/dl] } \\
\hline Before randomization & $164(11)$ & $159(10)$ \\
\hline After 12 weeks of treatment & $164(12)[0]$ & $142(10)[-11]^{\star \# \#}$ \\
\hline \multicolumn{3}{|l|}{ hsCRP [mg/l] } \\
\hline Before randomization & $2.2(0.5)$ & $2.0(0.5)$ \\
\hline After 12 weeks of treatment & $2.1(0.5)[-5]$ & $1.2(0.3)[-40]^{\star \star \# \#}$ \\
\hline \multicolumn{3}{|l|}{ TNF- $\alpha$ release $[\mathrm{pg} / \mathrm{ml}]$} \\
\hline Before randomization & $808(102)$ & $823(112)$ \\
\hline After 12 weeks of treatment & $821(120)[2]$ & $595(93)[-28] * \star \# \#$ \\
\hline \multicolumn{3}{|l|}{ Interleukin-1 $\beta$ release $[\mathrm{pg} / \mathrm{ml}]$} \\
\hline Before randomization & $85.2(12.1)$ & $82.1(11.0)$ \\
\hline After 12 weeks of treatment & $84.0(13.5)[-2]$ & $64.2(9.2)[-22]$ \\
\hline \multicolumn{3}{|l|}{ Interleukin-6 release $[\mathrm{ng} / \mathrm{ml}]$} \\
\hline Before randomization & $6.1(0.8)$ & $6.2(0.9)$ \\
\hline After 12 weeks of treatment & $6.3(1.0)[3]$ & $4.7(0.6)[-24]$ *\# \\
\hline \multicolumn{3}{|l|}{ MCP-1 release [ng/ml] } \\
\hline Before randomization & $14.2(1.8)$ & $14.6(2.0)$ \\
\hline After 12 weeks of treatment & $14.4(1.9)[1]$ & $11.2(1.8)[-23]$ \\
\hline
\end{tabular}

${ }^{1}$ Only data of subjects who completed the study were included in the final analyses. ${ }^{*} p<0.05,{ }^{* * *} p<0.01,{ }^{* * *} p<0.001 \mathrm{vs}$. before randomization; ${ }^{\#} p<0.05,{ }^{\# \#} p<0.01,{ }^{\# \# \#} p<0.001$ vs. fenofibrate + placebo group 
and cytokine release correlated with the reduction in HOMA-IR ( $\mathrm{r}$ values between 0.51 and $0.62, \mathrm{p}<$ 0.001 ) and in FFA ( $\mathrm{r}$ values between 0.34 and 0.41 , $\mathrm{p}<0.01)$. No other correlations were noted.

\section{Discussion}

In this study, we have found for the first time that high-dose metformin reduces monocyte cytokine release and decreases plasma hsCRP in fenofibratetreated patients with IGT. Although, combination therapy with metformin and fenofibrate was only moderately more effective in reducing monocyte secretion of proinflammatory cytokines and hsCRP than fenofibrate + placebo, our results may be clinically important. Considering that even small differences in the studied markers may be related to a various risk of cardiovascular disorders [2, 14], combined administration of metformin and a fibrate may bring important clinical benefits to prediabetic patients at high cardiovascular risk.

It should be underlined that fenofibrate administered alone to patients with both overt type 2 diabetes and IGT exhibited strong monocyte-suppressing and systemic anti-inflammatory effects $[3,4,8,11]$. In the latter group of patients, these effects were observed even though fenofibrate-treated IGT patients did not follow any special dietary regimen [8]. In the present study, besides fibrate therapy, all participants complied with lifestyle modifications for at least 6 months, the result of which was the normal lipid profile on the day of allocation. It is possible that the impact of fenofibrate and non-pharmacological treatment on monocyte secretory function and systemic inflammation was stronger than that of fenofibrate alone. In line with this explanation, baseline cytokine release in this study was much lower than in untreated IGT patients, slightly lower than in fenofibrate-treated IGT patients and only moderately elevated if compared with healthy subjects participating in other studies $[3,8]$.

Although metformin potentiates monocyte-suppressing and systemic anti-inflammatory effects of fenofibrate, if administered alone, it seems to produce only small pleiotropic effects. In line with this hypothesis, in the present study, baseline monocyte cytokine release and plasma hsCRP levels were higher in IGT patients treated chronically with metformin than with fenofibrate. This hypothesis is also supported by our previous study demonstrating that the effect of metformin on monocyte cytokine release (and hemostasis) in patients with recently diagnosed type 2 diabetes was small compared with simvastatin or fenofibrate [11].

The presence of a correlation between fenofibrate + metformin-induced changes in cytokine release and in plasma hsCRP, and the changes in the markers of insulin resistance induced by the combination therapy suggests that the impact of metformin on monocyte secretory function and systemic inflammation may be a consequence of the improvement in insulin action. Similarly as in our previous study, in the present one cytokine release moderately correlated with plasma hsCRP at baseline and during treatment. This suggests that plasma hsCRP levels do not reflect precisely secretory function of human monocytes and the effects of hypolipidemic and antidiabetic agents on the release of proinflammatory cytokines.

The addition of high-dose metformin to micronized fenofibrate was well tolerated and associated with favorable changes in glucose homeostasis. Interestingly, fenofibrate administered alone improved glucose homeostasis in different groups of patients, including those with prediabetic states $[5,6,8]$, while statin therapy, despite its cardiovascular benefits, is associated with a slightly increased risk of development of diabetes [12]. Considering that IGT patients are at higher risk of a progression to overt diabetes $[10,15]$, our findings are an additional argument for the combination therapy with a fibrate and metformin in the prevention of the development of vascular complications and overt diabetes in IGT patients.

\section{Conflict of interest:}

The authors declare no financial interests.

\section{Acknowledgments:}

This work was supported by the statutory grant NN-1-070/06 of the Medical University of Silesia. The authors are thankful to Mrs. Jarosława Sprada for her excellent technical support.

\section{References:}

1. Hansson GK: Inflammatory mechanisms in atherosclerosis. J Thromb Haemost, 2009, 7, Suppl 1, 328-331.

2. Kher N, Marsh JD: Pathobiology of atherosclerosis - a brief review. Semin Thromb Hemost, 2004, 30, 665-672. 
3. Krysiak R, Gdula-Dymek A, Bachowski R, Okopień B: Pleiotropic effects of atorvastatin and fenofibrate in metabolic syndrome and different types of pre-diabetes. Diabetes Care, 2010, 33, 2266-2270.

4. Krysiak R, Gdula-Dymek A, Okopień B: Effect of simvastatin and fenofibrate on cytokine release and systemic inflammation in type 2 diabetes mellitus with mixed dyslipidemia. Am J Cardiol, 2011, 107, 1010-1018.

5. Krysiak R, Gdula-Dymek A, Okopień B: Hemostatic effects of bezafibrate and $\omega-3$ fatty acids in isolated hypertriglyceridemic patients. Pharmacol Rep, 2011, 63, 763-771.

6. Krysiak R, Handzlik G, Okopień B: Hemostatic effects of fenofibrate in patients with mixed dyslipidemia and impaired fasting glucose. Pharmacol Rep, 2010, 62, 1099-1107.

7. Okopień B, Krysiak R, Haberka M, Herman ZS: Effect of monthly atorvastatin and fenofibrate treatment on monocyte chemoattractant protein-1 release in patients with primary hypercholesterolemia. J Cardiovasc Pharmacol, 2005, 45, 314-320.

8. Okopień B, Krysiak R, Herman ZS: Effects of short-term fenofibrate treatment on circulating markers of inflammation and hemostasis in patients with impaired glucose tolerance. J Clin Endocrinol Metab, 2006, 91, 1770-1778.

9. Okopień B, Krysiak R, Kowalski J, Madej A, Belowski D, Zieliński M, Herman ZS: Monocyte release of tumor necrosis factor- $\alpha$ and interleukin- $1 \beta$ in primary type IIa and IIb dyslipidemic patients treated with statins or fibrates. J Cardiovasc Pharmacol, 2005, 46, 377-386.
10. Petersen JL, McGuire DK: Impaired glucose tolerance and impaired fasting glucose - a review of diagnosis, clinical implications and management. Diab Vasc Dis Res, 2005, 2, 9-15.

11. Pruski M, Krysiak R, Okopień B: Pleiotropic action of short-term metformin and fenofibrate treatment, combined with lifestyle intervention, in type 2 diabetic patients with mixed dyslipidemia. Diabetes Care, 2009, 32, 1421-1424.

12. Sattar N, Preiss D, Murray HM, Welsh P, Buckley BM, de Craen AJ, Seshasai SR et al.: Statins and risk of incident diabetes: a collaborative meta-analysis of randomised statin trials. Lancet, 2010, 375, 735-742.

13. Scarpello JH, Howlett HC: Metformin therapy and clinical uses. Diab Vasc Dis Res, 2008, 5, 157-167.

14. Tedgui A, Mallat Z: Cytokines in atherosclerosis: pathogenic and regulatory pathways. Physiol Rev, 2006, 86, $515-581$.

15. Twigg SM, Kamp MC, Davis TM, Neylon EK, Flack JR; Australian Diabetes Society; Australian Diabetes Educators Association: Prediabetes: a position statement from the Australian Diabetes Society and Australian Diabetes Educators Association. Med J Aust, 2007, 186, 461-465.

16. Wilson HM, Barker RN, Erwig LP: Macrophages: promising targets for the treatment of atherosclerosis. Curr Vasc Pharmacol, 2009, 7, 234-243.

Received: June 13, 2012; in the revised form: May 22, 2013; accepted: June 7, 2013. 